\title{
Metallurgical Properties of Thin Aluminum Films Deposited in Vacuum
}

\author{
By Hiroshi Yamamoto* and Genichi Kamoshita*
}

\begin{abstract}
Thin aluminum films are widely used in the production of IC (Integrated Circuit) and LSI (Large Scale Integration), and are connected to other kinds of films or wires in these systems. Therefore, the reliability of these systems greatly depends on the metallurgical properties of thin aluminum films. The present study is concerned with these metallurgical properties. The thin films used in this study are those vacuum deposited onto silicon single crystals and are $1 \sim 2 \mu$ in thickness.

The metallurgical properties of the films are greatly affected by the conditions of vacuum deposition. That is, as the residual pressure decreased and substrate temperature increased, the crystal grain size of deposited films increased, and the electric resistance and hardness of them decreased. Within the limit of this experiment, the residual pressure of $10^{-6}$ Torr and the substrate temperature of $360^{\circ} \mathrm{C}$ give soft and ductile films and result in high rupture strength of the interconnection.

After discussing the results above mentioned from a metallurgical viewpoint, it is concluded that soft and ductile films are essential for obsaining the highly reliable interconnections.
\end{abstract}

(Received September 14, 1970)

\section{Introduction}

The thin film technology is very important in the IC production. This paper is concerned with aluminum thin films used for LSI (Large Scale Integration).

The semiconductor IC which is suitable for active device circuits and the thin film IC which is suitable for passive device circuits ${ }^{(1)}$ are combined together and this combination is named hybrid LSI. For this purpose, it is necessary to bond semiconductor IC chips to thin film circuits which are commonly formed on the alumina substrate. This bonding technology is classified as follows :

(1) Wire bonding. The terminals on the chip which is face-up-bonded onto the substrate and the terminals on the substrate are interconnected with fine lead wires.

(2) Wireless bonding. The terminals on the chip and the terminals on the substrate are directly interconnected, In this case, the chip must be face-down-bonded onto the substrate.

Recently, the method (2) is widely used for the production of the hybrid LSI in order to simplify the bonding processes, increase the operation efficiency and improve the reliability of products. There are three main technologies in this method, that is, the beam lead bonding ${ }^{(2)}$, the ball bonding ${ }^{(3)}$ and the ultrasonic flipchip-bonding. Our study was done as a part of the development of the ultrasonic flip-chip-bonding technology.

The property of materials to be mutually interconnected has to be fairly good, in order to get high strength of the interconnection. Therefore, in this study, metallurgical properties of the thin aluminum films were investigated for various conditions of vacuum deposition.

\section{Outline of the ultrasonic flip-chip- bonding}

An example of LSI modules is shown in Photo. $1^{(4)}$.

* Central Research Laboratory, Hitachi., Ltd., Kokubunji, Tokyo, Japan.

(1) E. Sugata : Denki Tsushin Gakkai Shi, 49 (1966), 597.

Trans. J IM
The substrate has 10 chips bonded on its surface.

The chip is about $1.5 \mathrm{~mm}$ square and has 16 terminals which are called pads. The chip is bonded onto the substrate ultrasonically as shown in Fig. 1. The pads are directly joined to the terminals, which are called pedestals, on the substrate. This technology is called the ultrasonic flip-chip-bonding.

The reliability of this LSI may greatly depend on the strength of the interconnections between pads and pedestals, and this strength is affected by the metallurgical properties of pads and pedestals.

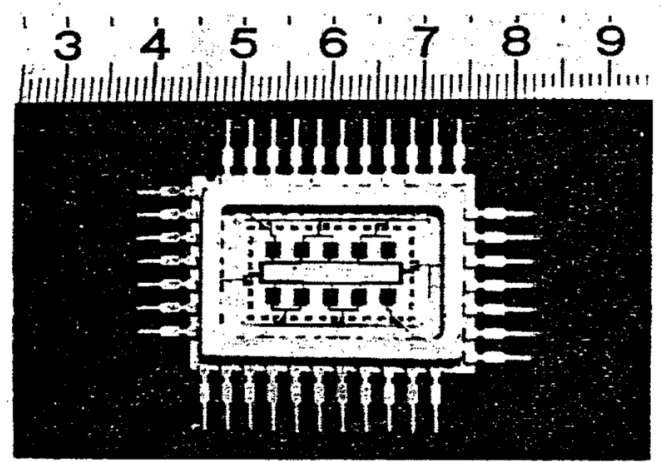

Photo. 1 ISI module.

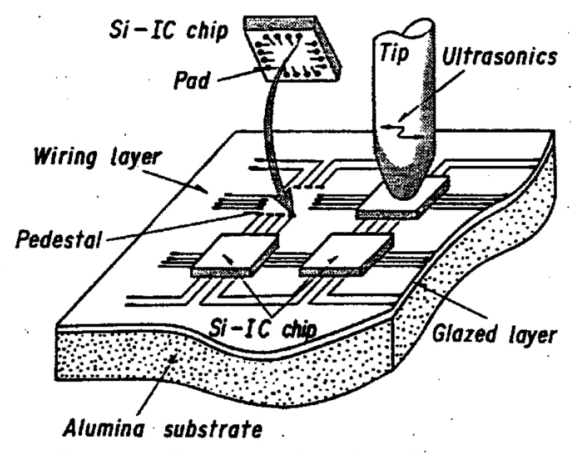

Fig. 1 Ultrasonic flip-chip-bonding.

(2) M.P.Lepselter : The Bell System Technical Journal, Oct. /Nov. (1966), 299

(3) A special issue on ASLT, IBM J., 11 (1967), 86.

(4) Research and Development, Hitachi Review, 19 (1970), 75.

1971 Vol. 12 


\section{Experimental Procedure}

The thin aluminum films were formed on the silicon wafers in the vacuum deposition appraratus as shown in Fig. 2. The substrate of silicon wafer was placed on the heater plate of sintered carbon. Then, aluminum was evaporated from a tungsten filament and deposited onto the silicon substrate. The deposition rate was about $450 \AA / \mathrm{sec}$, and the substrate temperature and the residual pressure were varied. The thickness of aluminum films were 8000 to $10000 \AA$. The light reflectivity, and the ratio of electric resistance at room temperature to that at the temperature of liquid nitrogen of these films were measured and the surface structure was studied. The hardness was measured for the film which was about $2 \mu$ thick.

The silicon wafer with the thin aluminum film was cleaved to make small chips. Before cleaving, the aluminum film was photo-etched to make the pads, 16 to each chip. Ten of these chips were flip-chip bonded onto the substrate to form the LSI module. Bonding strength was measured by the method shown in Fig. 3, that is, while keeping the substrate fixed, the chip was

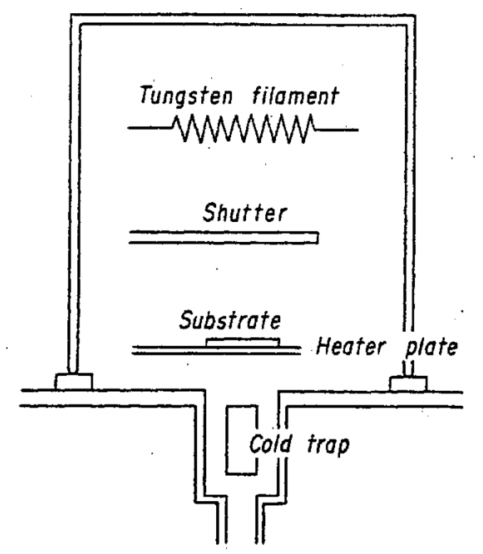

Fig. 2 Schema of the vacuum deposition apparatus. pushed parallel to the substrate surface with :a blade until the pad-pedestal interconnection was ruptured. This rupture strength was regarded as the bonding strength.

All the properties of the thin films mentioned above were examined for various conditions of vacuum deposition.

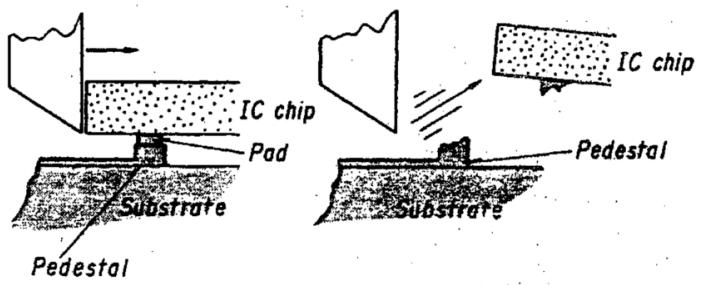

Fig. 3 Test of rupture strength for the estimation of bonding strength.

\section{Surface}

Electron micrographs of film surfaces are shown in Photo. 2. Ranks correspond to various temperatures and files to various pressures. From these photographs, it is known that the higher the temperature the larger the grain becomes and the more deeply is grooved the grain boundary. The grain boundary grooving brings rough surface. The higher the residual pressure, the rougher the surface becomes, too, as known from the appearance of a great number of hillocks.

The reflectivity, which is defined by the ratio of the intensity of reflected light to that of incident light on the surface of thin films deposited at various substrate temperatures and residual pressured, was measured and the result is shown in Fig. 4. Ultra-violet light with a wave length of $300 \AA$ was used in this experiment. The reflectivity shows a tendency to decrease as the substrate temperature increases, and this tendency is greater when residual pressure is lower. The above-mentioned result is certainly caused by the boundary grooving. Films

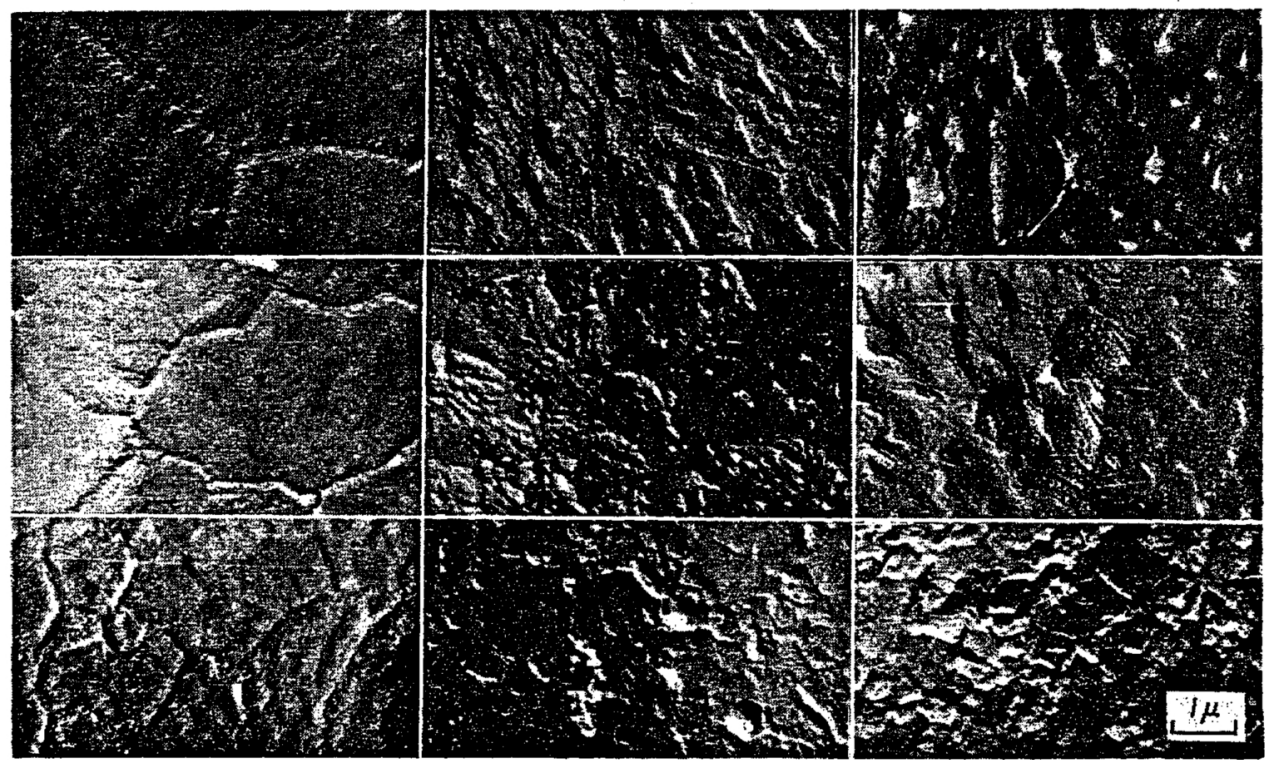

Photo. 2 Surface structure of thin aluminum films deposited at various residual pressures and substrate temperatures. Residual pressures are $0.5 \sim 1.2 \times 10^{-5} \mathrm{Torr}$ (left file), $5 \sim 7 \times 10^{-5}$ Torr (centre file) and $9 \sim 13 \times 10^{-5} \mathrm{Torr}$ (right file), and substrate temperatures are $360^{\circ} \mathrm{C}$ (top rank), $320^{\circ} \mathrm{C}$ (second rank) and $280^{\circ} \mathrm{C}$ (bottom rank). 


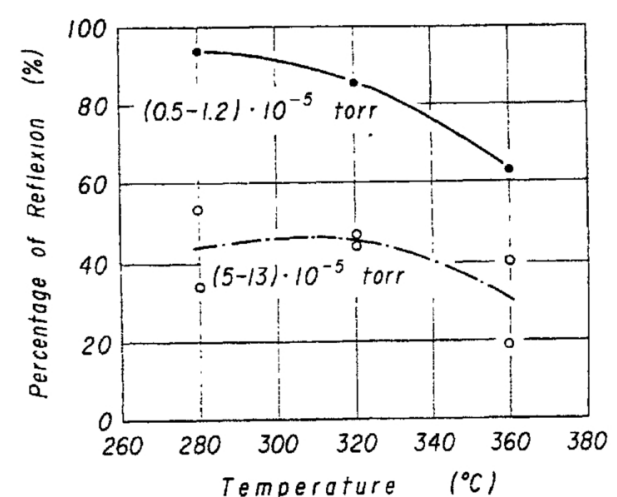

Fig. 4 Relation between the surface states and the deposition conditions of thin aluminum films. The surface state is represented by the reflexion for the light of 3000 angstroms in wave length, and deposition conditions are varied in both the residual pressure and the substrate temperature.

deposited in a low vacuum of the order of $10^{-4}$ Torr have a remarkably low reflectivity, too.

Heating the substrate at a rather high temperature and maintaining the vacuum fairly high during deposition cause the film surface to become fairly rough and reduce the reflectivity. However, the surface roughness in this case is different from that caused by low vacuum. The former does not seem to exert a serious ill-effect on the bonding.

\section{Grain growth}

From the electron micrographs of Photo. 2 the relation between grain size and substrate temperature was obtained, and is shown in Fig. 5. In this figure, closed circles are data for the deposition in higher vacuum and open circles are for those in lower vacuum.

The growth rate of grains was obtained from the above data and its logarithmic values are plotted against the inverse absolute substrate temperature as shown in Fig. 6. The plots for the same residual pressure lie on a nearly straight line. The apparent activation energy calculated from this slope is $10 \sim 20 \mathrm{kcal} / \mathrm{mol}$. This value seems to be smaller than the activation energy of self-diffusion of aluminium ${ }^{(5)}$. At a higher pressure of

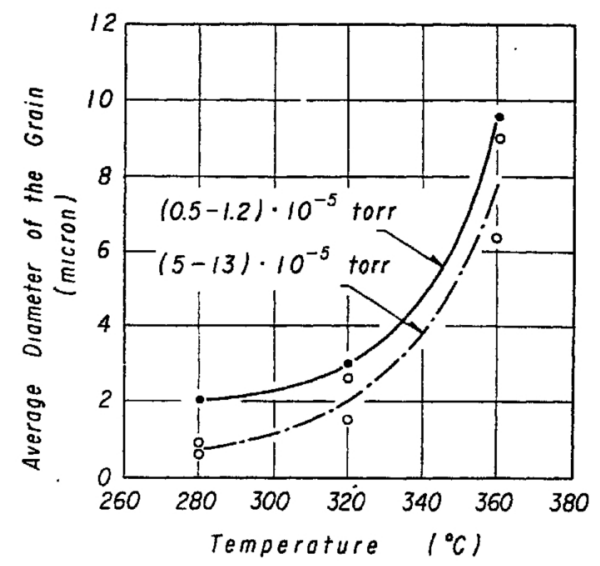

Fig. 5 Relation between the grain size and the deposition condition of thin aluminum film. The condition is varied in both the residual pressure and the substrate temperature.

(5) C. J. Smithels : Metal Reference Book, 2 (1955), 552.

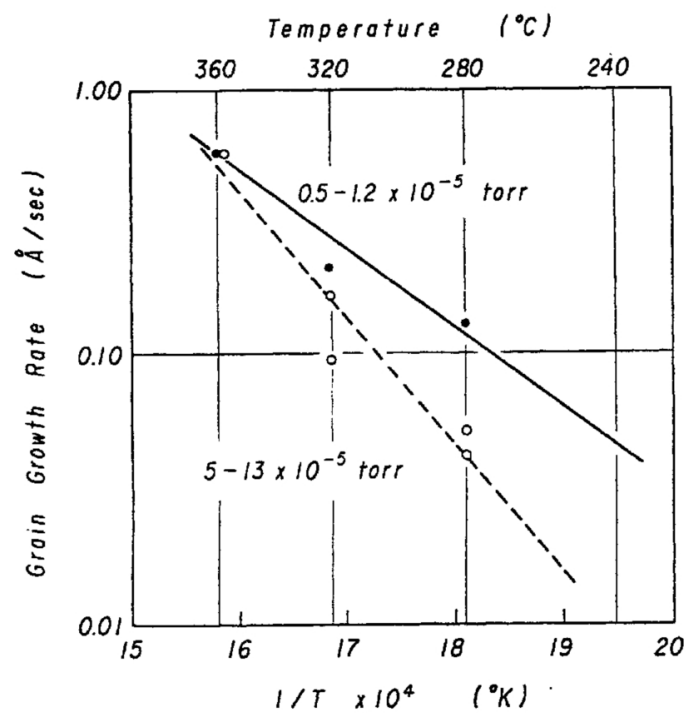

Fig. 6 Logarithmic grain growth rate of thin aluminum films versus reciprocal of the substrate temperature.

the order of $10^{-4}$ Torr, the growth rate is reduced. It is considered that the residual gas, especially oxygen, forms some oxide films around the aluminum grains and barriers for mutual diffusion of aluminum atoms.

\section{Hardness and the resistance ratio}

The hardness of the films deposited at various substrate temperatures and residual pressures during deposition was measured with the Knoop tester which had the measuring load of $0.5 \mathrm{~g}$. The result is shown in Fig. 7, that is, using higher temperature and higher vacuum produces soft and ductile films.

Figure 8 shows the relation between the ratio of the electric resistance at room temperature to that at the temperature of liquid nitrogen and the conditions of vacuum deposition, that is, substrate temperature and pressure. It is clear that the higher the substrate temperature and the vacuum, the higher the resistance ratio of the deposited film.

Generally, lattice defects and impurities increase the hardness and decrease the resistance ratio in bulk metals. It is considered that these relations are also applicable to

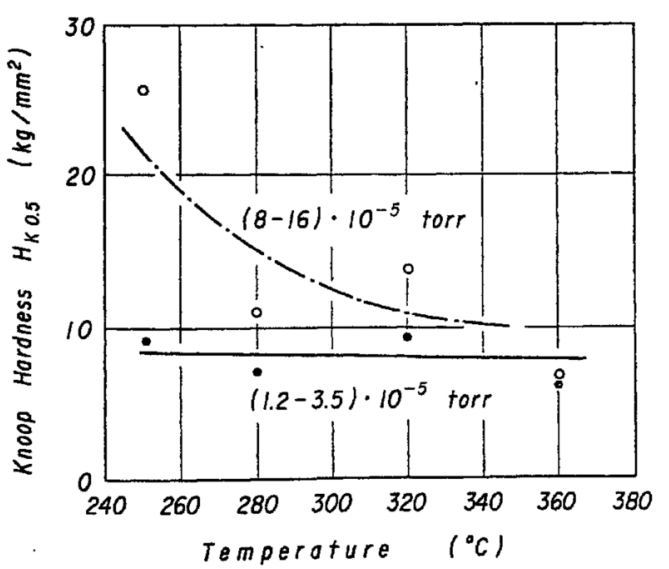

Fig. 7 Relation between the hardness and deposition condition of thin aluminum films. The condition is varied in both the residual pressure and substrate temperature. The hardness is measured with the Knoop tester of load 0.5 grams. 


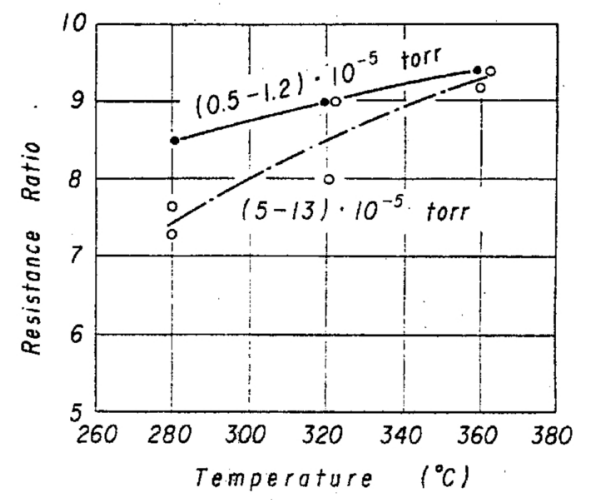

Fig. 8 Relation between the resistance ratio and the deposition condition. The condition is varied in both the residual pressure and the substrate temperature. The ratio is equal to (resistance at $\left.295^{\circ} \mathrm{K}\right) /\left(\right.$ resistance at $\left.77^{\circ} \mathrm{K}\right)$.

thin films. Lattice defects and impurities in thin films decrease as the substrate temperature increases and the residual pressure decreases, because lattice defects are annealed out by heating the substrate, and the collision probability and the sticking coefficient of the residual gas against the films are reduced by maintaining high vacuum and by heating the substrate at relatively high temperature.

Anyway, it seems quite reasonable that thin films deposited in high vacuum and at relatively high substrate temperature are ductile and have a rather high resistance ratio.

\section{Bonding strength}

The bond strength between the chip and the substrate was measured by the method shown in Fig. 3. This is the total strength of 16 bumps belonging to one chip, and is shown in Fig. 9 for various pressures and substrate temperatures. The result clearly shows that in high vacuum and at high substrate temperature good metal films are obtained, and films obtained in this way bring about a strong interconnection by the ultrasonic bonding.

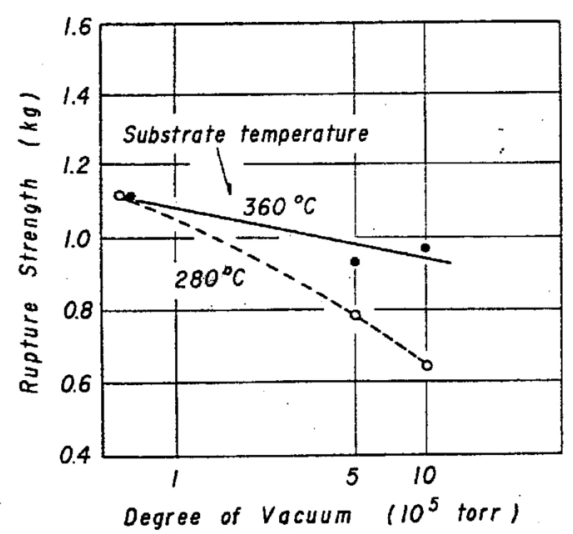

Fig. 9 Relation between the rupture strength of a pad-pedestal interconnection and the deposition condition of thin aluminum films. The condition is varied in both the residual pressure and the substrate temperature.

\section{Structure of the interface between pad and pedestal}

Photo. 3 is a electron micrograph of a vertical cross

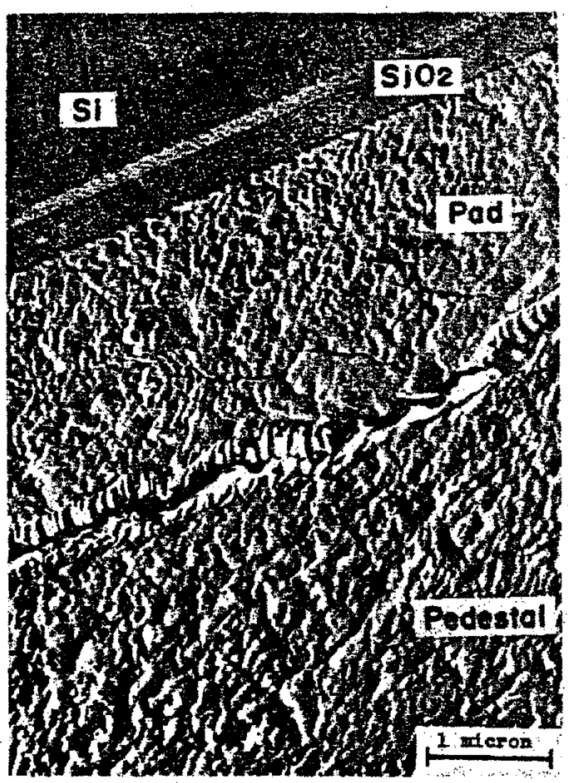

Pholo. 3 Vertical cross section of a pad-pedestal interconnection. (By courtesy of Mr. M. Hirano)

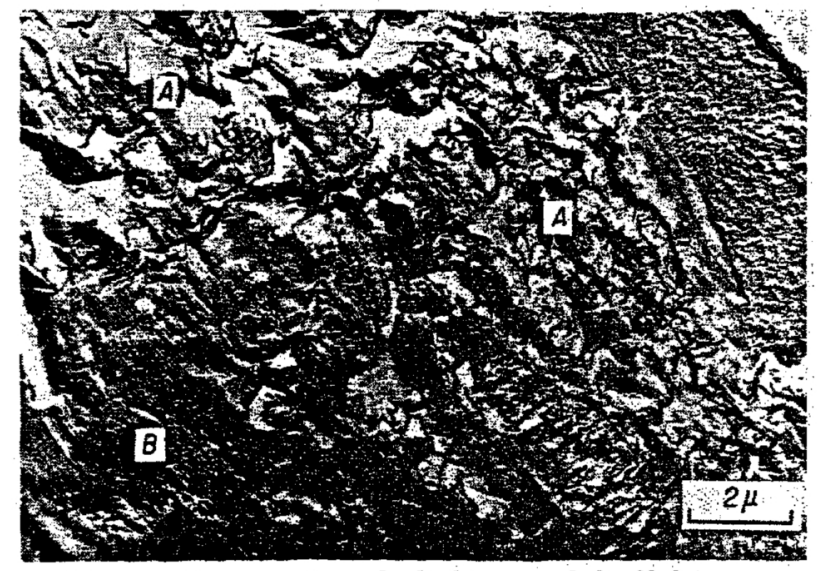

Photo. 4 Surface of a pad which was peeled off from an interconnection with pedestal. (By courtesy of Mr. M. Hirano)

section between a pad and a pedestal. In this picture, a thick irregular line is the interface and appears as a deep groove. In this photograph, only the structure of thin layers from the pad-pedestal interface is changed. These structures seem to be dendrites grown vertically from the interface. If it is valid, it is supposed that the surfaces of pad and pedestal melted by friction and were solidified very soon, forming the dendrite structure. Therefore, in order to complete this joining, it is necessary that a pad and a pedestal are fairly smooth on the surfaces for an optimum amount of friction.

The surface of a pad which was pulled off from a pedestal discloses the structure as shown in Photo. 4. In this structure, the parts which are very rough and denoted by $A$ are supposed to be the areas where the bonding is good (metallic bond) and to have the pattern of ductile fracture (cup-and-cone). On the contrary, the comparatively smooth part which has innumerable fine lines running parallel to each other and denoted by $\mathrm{B}$ was not actually bonded to the pedestal surface but was only in contact with it. These fine parallel lines had 
been marked by the surface of pedestal vibrated ultrasonically.

When the pad-pedestal interconnection was ruptured after a cyclic thermal shock of repeated heating at $200^{\circ} \mathrm{C}$ and cooling at $-70^{\circ} \mathrm{C}$, there appeared on the pad surface two kinds of structure as shown in Photo. 5. One is an irregular structure indicated by a mark $A$ on the photograph of Photo. 5, the other a polycrystalline structure marked B. The latter part of the pad was not actually bonded to the pedestal but only in contact with it in spite of the bonding operation and corresponds to the part having fine parallel lines in Photo. 4. Therefore, it is evident that the recrystallization occurred during the cyclic thermal shocks.

The cyclic thermal shock of ten initiates and propagates cracks in the interconnected part. An example of this crack is shown in Photo. 6 and is pointed by an arrow mark. To prevent cracks initiating and propagating, it is necessary for the pad and the pedestal to be ductile.

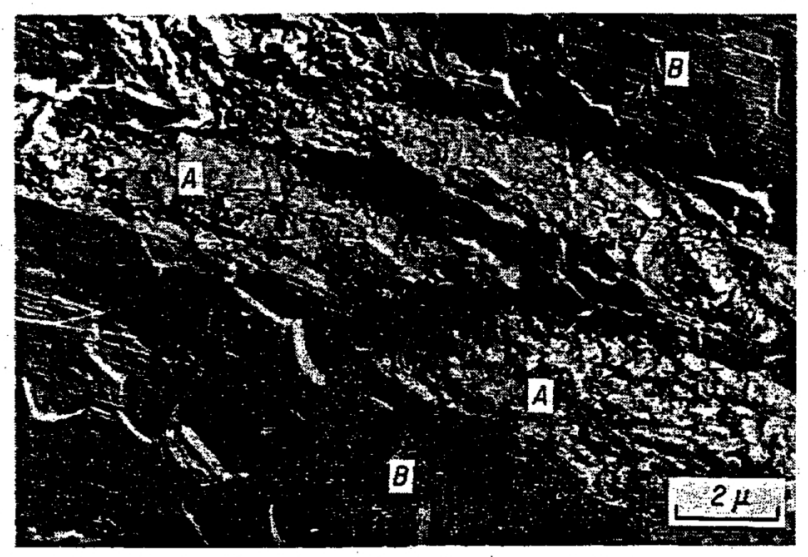

Photo. 5 Surface of a pad which was. peeled off from an interconnection with pedestal after suffered the cyclic thermal shock. (By courtesy of Mr. M. Hirano)

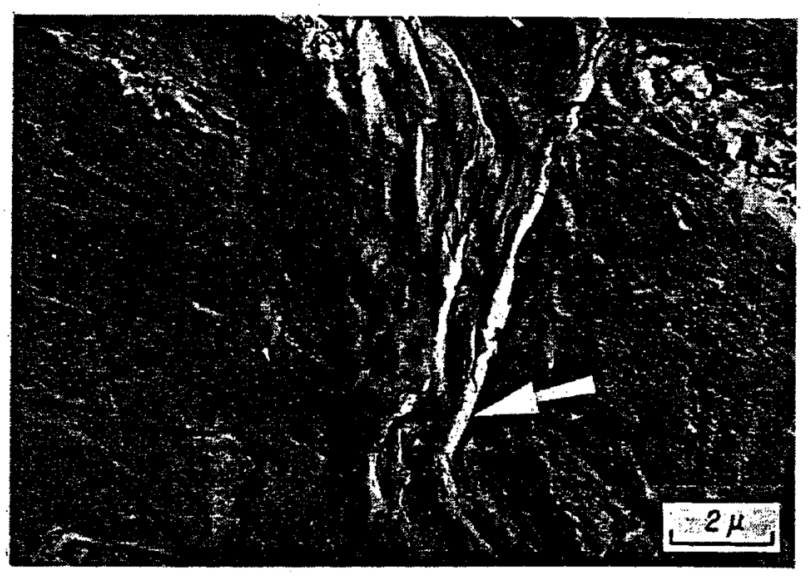

Photo. 6 Pad surface on which a crack initiated and propagated during the cyclic thermal shock. (By courtesy of Mr. M. Hirano)

\section{Discussion}

A film deposited from vapor may include a small amount of gas. Therefore, the solute gas in the film increases as the residual pressure increases. The quantity of the solute gas is too small to be detected by chemical analysis. The solute gas increases the electric resistance of the metal, but lattice defects have the same effect as the solute gas $^{(6)(7)}$. Therefore, it is actually impossible to know the gas content by measuring the electric resistance.

On the other hand, the residual gas in low vacuum may produce a very thin contamination layer on the film surface. As shown in this experiment (Fig. 6), the grain growth rate of deposited films is reduced at a relatively high residual pressure. This fact suggests that a thin contamination layer was formed over the surface of a crystal grain and disturbs diffusion from grain to grain. This contamination layer will cover the whole surface of the film. But the gas quantity or the thickness of this layer is undetectable by a usual technique such as X-ray analysis.

Residual gas gives a deposited film some chemical adsorption layer and solute impurities. When the substrate is heated during deposition, the sticking coefficient of gas will decrease and solute gas in a deposited film will decrease, too. However, chemical adsorption or oxidation will easily occur on the film surface at high temperature, if the vapor pressure of the adsorption layer or the oxide layer is very low.

Thin films formed by evaporation have great many lattice defects. According to several reports ${ }^{(8)(9)}$, there are $10^{10}$ to $10^{11}$ dislocations per unit area and about $10^{10}$ stacking faults $(10)$ per unit area in thin metal films. These numbers are larger by a factor of $10^{2}$ to $10^{4}$ than those of fully annealed bulk metal. Therefore, thin films are harder and have higher rupture strength and lower rupture strain ${ }^{(11)}$ than bulk metals. It is supposed that $10^{10}$ to $10^{11}$ dislocations per unit area in thin films are pinned down by the surface, impurities and/or jogs, preventing them migration and multiplication. Although the above facts are based on films thinner than several thousand $\AA$, the facts seem to be applicable to films about $1 \mu$ thick as well. Then, in the case of thin gold films, annihilation of stacking faults at $700^{\circ}$ to $800^{\circ} \mathrm{C}$ has been reported $^{(11) \sim(13)}$. Therefore, lattice defects in thin films are supposed to be annealed out by heating the substrate during deposition, and the films become soft and ductile.

If there are no contamination layers on the surfaces of metals to be joined, the interface of the joint will be a crystal grain boundary. However, since there may be a thin contamination layer of oxides or adsorbed gases, it is difficult to get a perfect interconnection. To acomplish the interconnection, the contamination layer must be broken and the pure metal surface must be exposed. This is realized by the ultrasonic vibration in the present experiment, and it is easily presumed that the thinner

(6) P. Jongenburger : Phys. Rev., 90 (1953), 710.

(7) A.H.Cottrell : Dislocations and Plastic Flow in Crystals, (1953), p. 153.

(8) J. W. Matthews : Phil. Mag., 4 (1959), 1017.

(9) G. A. Bassett, J. W. Menter and D. W. Pashley : Proc. Roy. Soc. A 246 (1958), 345.

(10) V. A. Phillips : Phil. Mag., 5 (1960), 571.

(11) D. W. Pashley : Proc. Roy. Soc., A 255 (1960), 218.

(12) K. Kimoto and Y.Fukano : Annual Meeting of Physical Society of Japan, 15 th $(1960), 3,11$.

(13) D. Watanabe : Annual Meeting of Physical Society of Japan, $18 \operatorname{th}(1963), 4,6$. 
the contamination layer and the more ductile the metal film, the better the interconnection will be. Now, it is impossible to know the thickness of the contamination layer, but some information can be obtained by measuring resistance and hardness. Furthermore, from this measurement, the ductility inside the film can be known indirectly. It is quite reasonable that a high bonding strength is obtained by ultrasonics for the film which has the high resistance ratio and low hardness. These properties are, as shown in this experiment, brought through by low residual pressure and high substrate temperature.

The reliability of a bonded chip is generally measured through the thermal shock test. The shock often gives the crack to a pad-pedestal interconnection, as shown in this experiment (Photo. 6). The fragments of very thin contamination layer are present in the interconnected part and play a role of notches when a thermal shock is applied. Further, dislocations in hard and brittle metal films will pile up at the edge of a notch and bring the initiation and propagation of cracks. Therefore, in order to get high reliability, films must be deposited in fairly high vacuum and at a rather high substrate temperature.

\section{Conclusion}

From the facts above mentioned, the following are concluded :

(1) Relatively ductile aluminum films can be deposited at low residual pressure (less than about $10^{-5}$ Torr) and at a high substrate temperature (about $360^{\circ} \mathrm{C}$ ), with the deposition rate fixed at about $450 \AA / \mathrm{sec}$.

(2) These ductile films are supposed to contain very small amounts of solute gases and lattice defects.

(3) A higher residual pressure (over about $10^{-4}$ Torr) may produce some contamination layer in grain boundaries and on the film surface.

(4) When a thin aluminum film is bonded to another aluminum film ultrasonically, the presence of a contamination layer and the non-ductility of the films (which are deposited at higher pressure and at low substrate temperature) do not give any good metallic interconnection, and may not guarantee high reliability.

(5) For good films deposited at the pressure of about $10^{-5}$ Torr and at the substrate temperature of $360^{\circ} \mathrm{C}$, a fairly high bonding strength more than $1.0 \mathrm{~kg}$ (per chip) is obtained.

\section{Acknowledgment}

The authors thank Mr. Mikio Hirano, Mr. Kazuyoshi Ueki and Dr. Seiichi Iwata for stimulating discussion. 